\title{
Evaluation of serum melatonin levels in preeclampsia - a case control study
}

\author{
Mahjabin, Rachna Agarwal, Rajarshi Kar, Alpana Singh, Richa Aggarwal \\ Corresponding author: Dr. Mahjabin, Postgraduate Student, Department of Obstetrics and \\ gynaecology, University College of Medical Sciences and GTB Hospital, Delhi, India; \\ Email : mahjabin1889@gmail.com
}

Distributed under Attribution-Non Commercial - Share Alike 4.0 International (CC BY-NC-SA 4.0)

\section{ABSTRACT}

\begin{abstract}
Aim: This study aimed to evaluate the association of serum melatonin levels in term pre-eclampsia (PE) subjects and compare it with normotensive healthy term pregnant women. Methodology: The study was conducted between November 2018 and April 2020. As per sample size calculation, 50 cases and 50 controls were enrolled in the study, matched for age, race and gestational age. Ethical clearance was obtained from Institutional Ethical Committee for human research. Night time (2 am) samples were taken. Analysis was done by ELISA test. Day time samples were also taken in cases to compare day and night time serum melatonin levels. Results: Mean value of serum melatonin level was lower in patients with preeclampsia $(93.18 \pm 61.5 \mathrm{pg} / \mathrm{mL})$ in Indian population as compared to controls $(109.18 \pm 69.86 \mathrm{pg} / \mathrm{mL})$, though not statistically significant $(\mathrm{p}=0.446)$. On plotting ROC curve for predicting severe PE we found that melatonin level with cut off of $96.9 \mathrm{pg} / \mathrm{mL}$ had AUC of 0.88 and was statistically significant. Severe PE $(47.32 \pm 26.88 \mathrm{pg} / \mathrm{mL})$ had significantly $(\mathrm{p}=0.001)$ lower serum melatonin level when compared to non-severe PE $(123.76 \pm 59.16 \mathrm{pg} / \mathrm{mL})$. Significantly lower value of melatonin was observed $(\mathrm{p}<0.01)$ in HELLP variety of PE. No significant difference was seen in melatonin levels between early and late onset PE. Conclusion: PE had lower levels of melatonin compared to controls and severe PE even had significantly lower melatonin levels compared to nonsevere PE and controls.
\end{abstract}

Keywords: Pre-eclampsia, melatonin.

Although the pathogenesis of preeclampsia is still unclear, it is well documented that placental dysfunction and oxidative stress contribute to the pathogenesis of preeclampsia ${ }^{1,2}$. Melatonin, a hormone produced by the pineal gland at night, plays an important role in the regulation of biological circadian rhythms, including blood pressure and sleep. In normal pregnancy during late trimester, maternal night time melatonin levels are significantly higher than nonpregnancy night values and this increase may be due to elevated pineal gland activity via the action of an unidentified placental hormone ${ }^{3}$. Melatonin is multifaceted with direct free radical scavenging and indirect antioxidant activities in both the mother and the fetus ${ }^{4,5}$. There is currently significant interest in the possible role of melatonin in abnormally functioning placentae as found in preeclampsia and FGR (fetal growth restriction). Melatonin acts as an antioxidant and its levels have found to be reduced in pre-eclampsia in various studies ${ }^{6-11}$. Melatonin can be used as a therapy for treatment of pre-eclampsia along with studies showing safety profile for fetus. There is paucity of literature on pre-eclampsia in Indian subjects. Hence, we plan to study the role of serum melatonin level in preeclampsia in a tertiary care centre.

Aim: To evaluate the association of serum melatonin levels in term pre-eclampsia subjects and compare it with normotensive healthy pregnant women. Comparison of its levels was done in severe and non-severe variety of PE. Difference in levels of melatonin was also estimated between

Received: $6^{\text {th }}$ October 2020, Peer review completed: $25^{\text {th }}$ January 2021, Accepted: $3^{\text {rd }}$ March 2021.

Mahjabin, Agarwal R, Kar R, Singh A, Aggarwal R. Evaluation of serum melatonin levels in preeclampsia - a case control study. The New Indian Journal of OBGYN. 2022; 8(2): 195 - 99. 
early and late onset PE.

\section{Materials and methods}

This was a case control study conducted between November 2018 and April 2020 in the department of obstetrics \& gynaecology in collaboration with department of biochemistry. This study was conducted on a value of melatonin in plasma expressed as picogram (pg). Being a continuous receptor variable, the mean value reported in a previous study was considered for calculating the sample size. In the previous study, Bouchlariotou et $\mathrm{al}^{12}$, the melatonin level in preeclampsia and healthy controls were respectively $48.4 \pm 24.7$ vs $85.4 \pm 2.9 \mathrm{pg} / \mathrm{mL}$. In order to detect this difference the required sample size for $\alpha$-error at $5 \%$ and power of study at $90 \%$, the required sample size is $<10$ per group. But since we were dealing with a continuous variable in order to ensure normalcy of the data, the recommended minimum sample size was 30 . However, we saw that $95 \%$ confidence interval in previously reported study was very wide. As per sample size calculation, 50 cases and 50 controls were enrolled in the study of which 50 were women with singleton pregnancy with diagnosis of pre-eclampsia for termination of pregnancy (dates confirmed by first trimester scan/sure of dates) meeting the inclusion and exclusion criteria were enrolled as cases and all normal healthy pregnant women matched for age, race and gestational age were taken as controls. Ethical clearance was obtained from Institutional Ethical Committee for human research.

Inclusion criterion was the diagnosis of pre-eclampsia according to the definition of the ACOG Task Force on Hypertension in Pregnancy. ${ }^{13}$ According to Task Force, preeclampsia was defined as new onset hypertension with systolic blood pressure $\geq 140 \mathrm{mmHg}$ or diastolic blood pressure $\geq 90 \mathrm{mmHg}$ after 20 weeks of gestation measured on two occasions at least four hours apart with proteinuria in a previously normotensive female. Proteinuria is defined as 24 hours urine protein $\geq 300 \mathrm{mg} / 24$ hours $(1+$ or more by dipstick method) or pre-eclampsia in absence of proteinuria with new onset of thrombocytopenia, renal insufficiency, impaired liver function, cerebral or visual symptoms or pulmonary edema. Preeclampsia was classified as severe on the basis of systolic blood pressure of $\geq 160 \mathrm{mmHg}$, or diastolic blood pressure of $\geq 110 \mathrm{mmHg}$ or new onset of hypertension with any of the features like thrombocytopenia, impaired liver function, renal insufficiency, pulmonary edema or visual symptoms.

Exclusion criterion was pregnancy with anomalous fetus, known diabetic or gestational diabetes, unsure of dates, pregnancy with $\mathrm{Rh}$ isoimmunisation, chronic hypertension with superimposed preeclampsia, personal history of smoking, pregnancy with any chronic disease, chronic liver and renal disease, pregnancy conceived from assisted reproduction technology. Early onset PE was defined as onset of hypertension before 34 weeks and late onset PE as $>34$ weeks. Two $\mathrm{ml}$ of maternal plasma sample was taken in EDTA vial at night (at 2 a.m.) for all PE cases planned for termination of pregnancy and controls as per hospital protocol. Twenty day time samples of pre-eclampsia cases were also taken. Samples were stored at $-20^{\circ}$ C. Samples were analysed to measure serum melatonin concentrations by ELISA kit.

Determination of serum levels of melatonin: The plasma levels of melatonin in women with preeclampsia and normotensive pregnant women were measured using melatonin ELISA kit following manufacturer's instructions. The analytical sensitivity of melatonin ELISA kit is 1.6 $\mathrm{pg} / \mathrm{mL}$.

Statistical analysis: Categorical variables were presented in number and percentage (\%) and continuous variables were presented as mean $\pm \mathrm{SD}$ and median. Quantitative variables were compared using Mann-Whitney Test. Qualitative variables were correlated using Chi-Square test. Receiver operating characteristic curve was used to find out cut off point of melatonin levels for predicting cases and severe preeclampsia in cases. A $p$ value of $<0.05$ was considered statistically significant. The analysis was done using Statistical Package for Social Sciences (SPSS) version 21.0.

\section{Results}

Age, race and gestational age were matched for cases and controls. Maximum number of women in both the groups were between 20-30 years of age, housewives by occupation, hindu by religion, belonged to urban area. Patients belonged to same race and ethnicity. The $p$ value was found to be nonsignificant for all of the parameters in cases versus controls hence the two groups were matched, which has been tabulated in table 1 .

Mean BMI in cases was $25.9 \pm 2.62 \mathrm{~kg} / \mathrm{m}^{2}$ which was significantly higher $(\mathrm{p}=0.001)$ than controls i.e. $24.29 \pm 1.76$ $\mathrm{kg} / \mathrm{m}^{2}$. Out of 50 cases, 30 cases were non-severe PE and 20 cases were severe PE. Out of 20 severe pre-eclampsia, 5 cases were of HELLP (Hemolysis, Elevated liver enzymes, Low platelet count) syndrome. Among these cases of HELLP, 3 were partial with thrombocytopenia only and 2 were complete HELLP. 
The New Indian Journal of OBGYN. 2021 (January-June);8(2)

Table 1: Comparison of socio-demographic characteristics of the study groups

\begin{tabular}{|c|c|c|c|}
\hline Characteristics & $\begin{array}{l}\text { Group } 1 \\
\text { Cases }(n=50)\end{array}$ & $\begin{array}{l}\text { Group } 2 \\
\text { Controls }(\mathrm{n}=50)\end{array}$ & $\begin{array}{l}P \\
\text { value }\end{array}$ \\
\hline Age in yrs $($ mean \pm SD) & $26.18 \pm 2.94$ & $25.76 \pm 2.85$ & 0.744 \\
\hline Residential Area & $\%(\mathrm{~N})$ & $\%(\mathrm{~N})$ & \multirow{3}{*}{0.806} \\
\hline Urban & $78 \%(39)$ & $80 \%(40)$ & \\
\hline Rural & $22 \%(11)$ & $20 \%(10)$ & \\
\hline Socioeconomic status & & & \multirow{6}{*}{0.512} \\
\hline Class I (upper) & 0 & 0 & \\
\hline Class II (upper middle) & $10 \%(5)$ & $6 \%(3)$ & \\
\hline Class III (lower-middle) & $58 \%(29)$ & $52 \%(26)$ & \\
\hline Class IV (Upper-Lower) & $32 \%(16)$ & $42 \%(21)$ & \\
\hline Class V (lower) & 0 & 0 & \\
\hline Occupation & & & \multirow{3}{*}{0.249} \\
\hline Housewives & $90 \%(45)$ & $82 \%(41)$ & \\
\hline Employed & $10 \%(5)$ & $18 \%(9)$ & \\
\hline Religion & & & \multirow{3}{*}{0.123} \\
\hline Hindu & $78 \%(39)$ & $64 \%(32)$ & \\
\hline Muslim & $22 \%(11)$ & $36 \%(18)$ & \\
\hline
\end{tabular}

severe PE. Most common complication in severe PE was

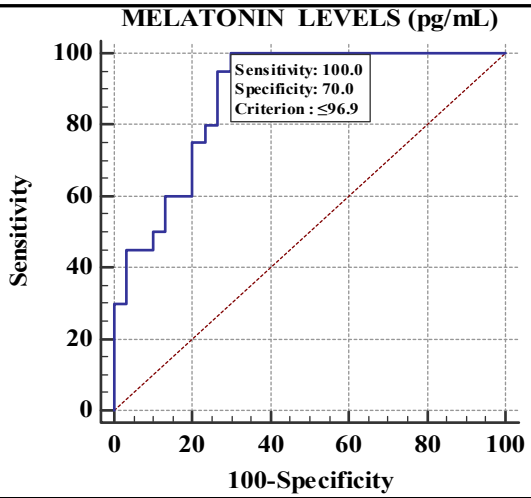

Figure 2: Receiver operating characteristic curve of melatonin levels for predicting severe preeclampsia showing $\mathrm{AUC}$ of 0.88 and $p<0.01$ with sensitivity and specificity $100 \%$ and $70 \%$ respectively.

found to be jaundice $(20 \%, \mathrm{n}=4)$ followed by renal failure
Table 2: Receiver operating characteristic curve of melatonin levels for predicting cases and severe preeclampsia in cases

\begin{tabular}{lll}
\hline $\begin{array}{l}\text { Melatonin levels } \\
\text { (pg/mL) }\end{array}$ & $\begin{array}{l}\text { For predicting } \\
\text { cases }\end{array}$ & $\begin{array}{l}\text { For predicting severe } \\
\text { preeclampsia }\end{array}$ \\
\hline Area under the ROC curve (AUC) & 0.544 & 0.88 \\
Standard Error & 0.0583 & 0.0465 \\
95\% Confidence interval & 0.442 to 0.644 & 0.757 to 0.955 \\
P value & 0.4484 & $<0.0001$ \\
Cut off & $\leq 153.2$ & $\leq 96.9$ \\
Sensitivity $(95 \%$ CI) & $92 \%(80.8-97.8 \%)$ & $100 \%(83.2-100.0 \%)$ \\
Specificity $(95 \%$ CI) & $24 \%(13.1-38.2 \%)$ & $70 \%(50.6-85.3 \%)$ \\
PPV (95\% CI) & $54.8 \%(43.5-65.7 \%)$ & $69 \%(49.2-84.7 \%)$ \\
NPV (95\% CI) & $75 \%(47.6-92.7 \%)$ & $100 \%(83.9-100.0 \%)$ \\
\hline
\end{tabular}

$(10 \%, n=2)$ and coagulation failure $(2 \%, n=1)$. Antenatally fetal growth restriction along with deranged doppler was noted in 7 cases, of which 5 had severe PE manifestations. Most of the PE cases $(n=41,82 \%)$ had induction of labour while rest delivered spontaneously. Caesarean section was done in 15 cases (30\%). There was no maternal mortality. No significant difference was seen when night time samples were compared with day time in PE cases. Neonatal weight of

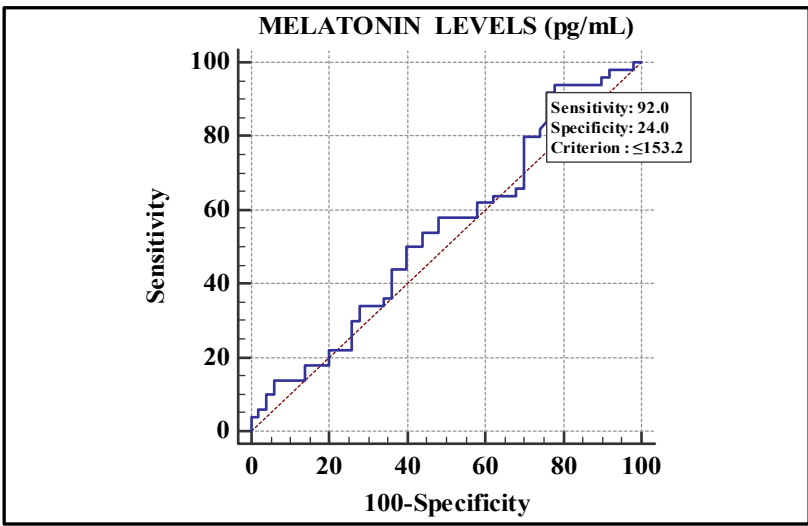

Figure 1: Receiver operating characteristic (ROC) curve of melatonin levels for predicting cases showing AUC of 0.54 with melatonin cut off $153.2 \mathrm{pg} / \mathrm{mL}$ and $\mathrm{p}$ value 0.44 with sensitivity and specificity $92 \%$ and $24 \%$ respectively.

There were 20 case of early onset PE of which 11 progressed to severe PE. Whereas in late onset PE, only 14 developed cases had low birth weight (mean $=2.5 \pm 0.3 \mathrm{~kg}$ ) when compare to controls $(2.62 \pm 0.28 \mathrm{~kg})$. Thirteen neonates were admitted in neonatal intensive care unit and we observed 4 neonatal deaths of which all were born to severe PE mother.

Serum melatonin levels were lower in cases than controls (93.18 $\pm 61.55 \mathrm{vs} 109.18 \pm 77.12 \mathrm{pg} / \mathrm{ml}, \mathrm{p}=0.446)$ although not statistically significant. Serum melatonin levels were significantly lower in severe PE cases than non-severe PE $(47.32 \pm 26.88$ vs $123.76 \pm 59.16 \mathrm{pg} / \mathrm{ml}, \quad \mathrm{p}=0.001)$. Day $(106.14 \pm 46.59 \mathrm{pg} / \mathrm{mL})$ and night time $(93.18 \pm 61.55 \mathrm{pg} / \mathrm{mL})$ melatonin levels were compared among cases and it was found to be non-significant. No significant difference was seen in melatonin levels between early and late onset PE. When we compared serum melatonin levels between HELLP and non-HELLP we found significantly lower value of serum melatonin level in HELLP and non-HELLP group $(24.8 \pm 12.92$ vs $100.78 \pm 60.06 \mathrm{pg} / \mathrm{mL}, \quad \mathrm{p}=0.001)$. On comparing FGR and non-FGR cases among PE cases, mean value of serum melatonin level among FGR and non-FGR 
was $(59.2 \pm 47.48 \mathrm{vs} 98.72 \pm 62.19 \mathrm{pg} / \mathrm{mL}, \mathrm{p}=0.134)$ was found to be non-significant.

We plotted two curves for predicting PE cases and severe PE category. Table 2 and figure 1 show that melatonin level for predicting PE cases had $\mathrm{AUC}(0.54)$ with melatonin cut off of $153.2 \mathrm{pg} / \mathrm{mL}$ and low specificity (24\%) and had no significance. Whereas table 2 and figure 2 shows melatonin level with cut off of $96.6 \mathrm{pg} / \mathrm{mL}$ had AUC of 0.88 and was statistically significant. On plotting ROC curve, among severe PE, all cases $(100 \%)$ had melatonin level $\leq 96.9$ $\mathrm{pg} / \mathrm{mL}$ while among non-severe PE, $70.00 \%$ had melatonin levels $>96.9 \mathrm{pg} / \mathrm{mL}$.

\section{Discussion}

Melatonin, a potent free radical scavenger of the highly toxic hydroxyl radical and other reactive oxygen species, increases the levels of several antioxidative enzymes and inhibits the pro-oxidative enzyme nitric oxide synthase. Preeclampsia is an oxidative stress related condition. Melatonin levels may be depressed because melatonin, as a radical scavenger, is being consumed very rapidly by the enhanced reactive oxygen species in this disorder. We studied role of melatonin in night time samples of cases and controls. As melatonin has diurnal variation, we analysed day and night melatonin in 20 cases of PE and found no difference. These findings also suggested by Bouchlariotou et $\mathrm{al}^{12}$ and Nakamura ${ }^{14}$. Hence taking night time value of serum melatonin in our study for all PE cases and controls is relevant.

Melatonin has been studied in literature by Bouchlariotou et al, Zeng et al and Nakamura et al and all have shown its level to be significantly decreased in PE cases vs controls. The melatonin level in Bouchlariotou et $\mathrm{al}^{12}$ study was $85.4 \pm 2.9 \mathrm{pg} / \mathrm{mL}$ in controls $48.4 \pm 24.7 \mathrm{pg} / \mathrm{mL}$ in cases. However, the melatonin level in our study was $93.18 \pm 61.55$ $\mathrm{pg} / \mathrm{mL}$ in cases and $109.18 \pm 69.86 \mathrm{pg} / \mathrm{mL}$ in controls. Severe PE cases had significantly lower $(47.32 \pm 26.88)$ melatonin level than non-severe PE (123.76 \pm 59.16$) \quad(\mathrm{p}=0.001)$. Nakamura et $\mathrm{al}^{14}$ and Zeng et a ${ }^{15}$ also had same observation of severe PE, had significantly lower value of melatonin than non-severe PE and controls. Another interesting finding was that severe PE category has even lower value than nonsevere PE and controls as evidenced by study of Nakamura ${ }^{14}$.

Nakamura had studied 19 cases of PE after 32 weeks of gestation and made 2 groups of 32-36 weeks and beyond 36 weeks and even studied umbilical artery and umbilical vein melatonin level. They suggested severe PE have significantly lower level $(50 \mathrm{pg} / \mathrm{mL}, \mathrm{p}=0.01)$ beyond 36 weeks of gestation in coherence with our study type. Zeng had studied melatonin level in various seasons and found no seasonal variation in melatonin during pregnancy. Zeng also found significantly lower values in PE cases $(42.75 \mathrm{pg} / \mathrm{mL})$ but had studied only day time samples. They had even related melatonin with onset of pregnancy and severity but found no relation. We found lower level in late onset PE but with no statistical significant. Hence we suggest that lowering of melatonin has linear correlation with severity.

We even analysed subgroups of severe PE cases like HELLP and other organ failure. The HELLP variety had significantly lower $(24.8 \pm 12.92 \mathrm{pg} / \mathrm{mL})$ melatonin than nonHELLP variety $(100.78 \pm 60.06 \mathrm{pg} / \mathrm{mL})$. This finding may possibly be explained as "failure of compensatory mechanism of melatonin acting as antioxidant and preventing severe $\mathrm{PE}^{\prime \prime}$. So melatonin below a particular value results in severe manifestations of $\mathrm{PE}$ especially HELLP. On plotting ROC curve for predicting severe PE, we found cut off of value of melatonin $96.6 \mathrm{pg} / \mathrm{mL}, \mathrm{AUC}$ as 0.88 with good sensitivity and specificity.

The strengths of this study were that this was a case control model study in a previously uninvestigated population from Indian subcontinent. The study had a prospective study design and all the subjects were matched for socio-demographic factors and obstetrical profile. Our study focused on a welldefined PE group excluding gestational hypertension and chronic hypertension cases to reduce ambiguity of pregnancy related hypertensive case definitions. We even analysed subgroup of severe PE i.e. HELLP category. Most of the published studies in literature have been done on western population ${ }^{14-16}$. Present study has been done in Indian setting comparing serum melatonin level among preeclampsia cases of varying severity and normal control. We tried to eliminate the bias by taking all samples beyond 36 weeks period of gestation.

The limitations of this study were limited number of cases. Immunohistochemistry, melatonin receptor studies were not a part of our study. Serum melatonin level in placenta and neonatal cord blood were not considered in our study due to limited resources. The result of only one hospital cannot be generalized for all settings.

\section{Conclusion}

PE had lower levels of melatonin compared to controls and severe PE even had significantly lower melatonin levels compared to non-severe PE and controls. Thereby lowered levels lead to severe manifestations in PE. It is concluded that in future there might be some possibility of melatonin drug acting as a therapeutic agent. Significantly lower value 
of serum melatonin level found in HELLP group when compared with non-HELLP group.

\section{Conflict of interest: None. Disclaimer: Nil.}

\section{References}

1. Hubel CA. Oxidative stress in the pathogenesis of preeclampsia. Proc Soc. Exp Biol Med. 1999; 222(3): 222-35.

2. Roberts JM, Hubel CA. Is oxidative stress the link in the two-stage model of pre-eclampsia? Lancet. 1999; 354(9181):788-9.

3. Kivela A. Serum melatonin during human pregnancy. Acta Endocrinol. 1991; 124(3): 233-7.

4. Miller SL, YawnoT, Alers NO, Castillo-Melendez M, Supramaniam VG, Vanzyl N, et al. Antenatal oxidant treatment with melatonin to decrease newborn neurodevelopmental deficits and brain injury caused by fetal growth restriction. J Pineal Res. 2014; 56(3); 283-94.

5. Miller SL, Yan EB, Castillo-Melendez $\mathrm{M}$, et al. Melatonin provides neuroprotection in the late gestational fetal sheep brain in response to umbilical cord occlusion. Dev Neuro sci. 2005; 27: 200-10.

6. Marseglia L, D’Angelo G, Manti S, Reiter RJ, Gitto E. Potential Utility of Melatonin in Preeclampsia, Intrauterine Fetal Growth Retardation and Perinatal Asphyxia. Reprod Sci. 2016; 23(8): 970-7.

7. GittoE, Pellegrino S, Gitto P, et al. Oxidative stress of the newborn in the pre and postnatal period and the clinical utility of melatonin. J Pineal Res. 2009; 46: 128-39.

8. Voiculescu S, Zygouropoulos N, Zahiu C, Zagrean A. Role of melatonin in embryo fetal development. J Med Life. 2014; 7(4): 488-92.

9. Aversa S, Pellegrino S, Barberi I, Reiter RJ, Gitto E. Potential utility of melatonin as an oxidant during pregnancy and in the perinatal period. J Matern Fetal Neonatal Med. 2012; 25(3): 207-21.
10. Robertson NJ, Faulkner S, Fleiss B, et al. Melatonin augments hypothermic neuroprotection in a perinatal asphyxia model. Brain. 2013; 136(pt 1): 90-105.

11. Aly H, Elmahdy H, El-Dib M, et al. Melatonin use for neuroprotection in perinatal asphyxia: a randomized controlled pilot study. J Perinatol. 2015; 35(3):186-91.

12. Bouchlariotou S, Liakopoulos V, Giannopoulou M, et al. Melatonin secretion is impaired in women with preeclampsia and an abnormal circadian blood pressure rhythm. Renal failure. 2014; 36(7):1001-7.

13. Hypertension in pregnancy. Report of the American College of Obstetricians and Gynecologists' Task Force on Hypertension in Pregnancy. Obstet Gynecol. 2013;122(5):1122-31.

14. Nakamura $Y$, Tamura H, Kashida S, Takayama H, Yamagata Y, Karube A, et al. Changes of serum melatonin level and its relationship to feto-placental unit during pregnancy. J Pineal Res. 2001; 30(1): 29-33.

15. Zeng K, Gao Y, Wan J, Tong M, Lee AC, Zhao M, et al. The reduction in circulating levels of melatonin may be associated with the development of preeclampsia. J Hum Hypertens. 2016; 30(11): 666-71.

\footnotetext{
Mahjabin $^{1}$, Rachna Agarwal ${ }^{2}$, Rajarshi Kar ${ }^{3}$, Alpana Singh ${ }^{4}$, Richa Aggarwal ${ }^{5}$

${ }^{1}$ Postgraduate Student, Department of Obstetrics and gynaecology, University College of Medical Sciences and GTB Hospital, Delhi, India; ${ }^{2}$ Professor, Department of Obstetrics and gynaecology, University College of Medical Sciences and GTB Hospital, Delhi, India; ${ }^{3}$ Assistant Professor, Department of Biochemistry, University College of Medical Sciences and GTB Hospital, Delhi, India; ${ }^{4}$ Associate Professor, Department of Obstetrics and gynaecology, University College of Medical Sciences and GTB Hospital, Delhi, India; ${ }^{5}$ Assistant Professor, Department of Obstetrics and gynaecology, University College of Medical Sciences and GTB Hospital, Delhi, India.
} 